\title{
Evolution of Mosquito-Based Arbovirus Surveillance Systems in Australia
}

\author{
Andrew F. van den Hurk, ${ }^{1}$ Sonja Hall-Mendelin, ${ }^{1}$ Cheryl A. Johansen, ${ }^{2}$ \\ David Warrilow, ${ }^{1}$ and Scott A. Ritchie ${ }^{3}$ \\ ${ }^{1}$ Public Health Virology, Communicable Diseases Unit, Queensland Health Forensic and Scientific Services, 39 Kessels Rd, \\ Coopers Plains, Queensland 4108, Australia \\ ${ }^{2}$ Discipline of Microbiology and Immunology, School of Biomedical, Biomolecular and Chemical Sciences, \\ The University of Western Australia, Nedlands, Western Australia 6009, Australia \\ ${ }^{3}$ School of Public Health, Tropical Medicine and Rehabilitative Services, James Cook University, Cairns, Queensland 4870, Australia
}

Correspondence should be addressed to Andrew F. van den Hurk, andrew_hurk@health.qld.gov.au

Received 2 November 2011; Accepted 7 December 2011

Academic Editor: Bradley J. Blitvich

Copyright (c) 2012 Andrew F. van den Hurk et al. This is an open access article distributed under the Creative Commons Attribution License, which permits unrestricted use, distribution, and reproduction in any medium, provided the original work is properly cited.

\begin{abstract}
Control of arboviral disease is dependent on the sensitive and timely detection of elevated virus activity or the identification of emergent or exotic viruses. The emergence of Japanese encephalitis virus (JEV) in northern Australia revealed numerous problems with performing arbovirus surveillance in remote locations. A sentinel pig programme detected JEV activity, although there were a number of financial, logistical, diagnostic and ethical limitations. A system was developed which detected viral RNA in mosquitoes collected by solar or propane powered $\mathrm{CO}_{2}$-baited traps. However, this method was hampered by trap-component malfunction, microbial contamination and large mosquito numbers which overwhelmed diagnostic capabilities. A novel approach involves allowing mosquitoes within a box trap to probe a sugar-baited nucleic-acid preservation card that is processed for expectorated arboviruses. In a longitudinal field trial, both Ross River and Barmah Forest viruses were detected numerous times from multiple traps over different weeks. Further refinements, including the development of unpowered traps and use of yeast-generated $\mathrm{CO}_{2}$, could enhance the applicability of this system to remote locations. New diagnostic technology, such as next generation sequencing and biosensors, will increase the capacity for recognizing emergent or exotic viruses, while cloud computing platforms will facilitate rapid dissemination of data.
\end{abstract}

\section{Introduction}

Over 75 different arboviruses have been isolated in Australia, with some being the aetiological agents of human disease [1]. Ross River virus (RRV) and Barmah Forest virus (BFV), both belonging to the genus Alphavirus, are responsible for the greatest number of annual disease notifications [2]. While Murray Valley encephalitis (MVEV) and Kunjin virus (KUNV; a subtype of West Nile virus (WNV)) are endemic in northern Australia, they can cause periodic outbreaks of acute encephalitis in southern and central regions. Dengue outbreaks occur regularly in northern Queensland, the only region in Australia where Aedes aegypti, the primary vector of dengue viruses (DENVs), occurs [3]. When Japanese encephalitis virus (JEV) emerged in the mid-1990s in the Torres Strait and Cape York Peninsula (Figure 1), it was feared that it would become a serious public health issue on the Australian mainland [4]. Finally, as competent vectors are present, there is always the potential for exotic arboviruses, such as the North American strain of WNV, chikungunya virus (CHIKV), and Rift Valley fever virus, to be introduced into Australia [5-7].

There is a need for informed decisions to be made regarding the implementation of control strategies for both endemic and exotic arboviruses. A comprehensive surveillance strategy is essential to ensure that elevated or emergent virus activity is detected before an outbreak occurs, as well as for establishing a baseline of arbovirus activity. 


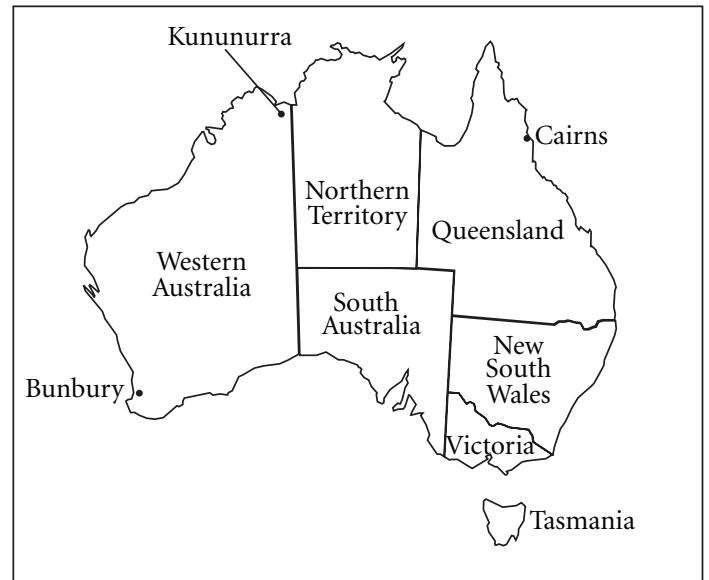

(a)

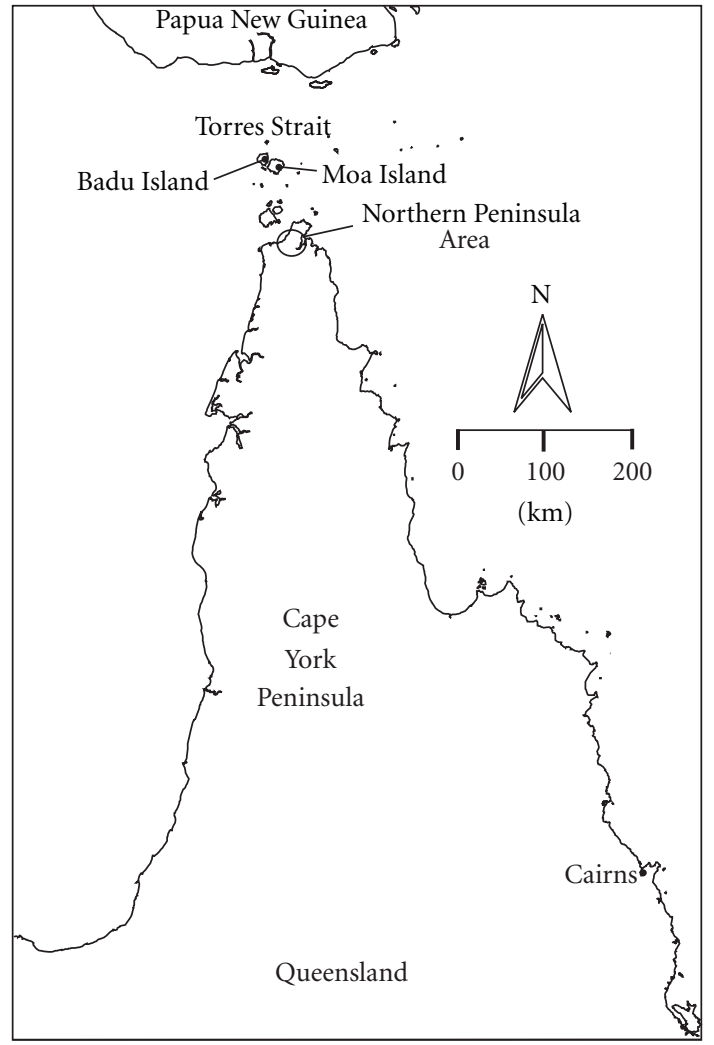

(b)

Figure 1: Map of (a) Australia and (b) northern Queensland showing locations mentioned in the text.

Together with meteorological and vertebrate host data, this may then facilitate accurate prediction of virus fluctuations and outbreaks. The relevant authorities can use the data obtained from such strategies to formulate control initiatives, including vaccination, mosquito control, and/or public education. The mainland Australian states maintain proactive mosquito and sentinel animal-based arbovirus surveillance programmes, which are primarily used to detect elevated RRV and MVEV activity. Outbreaks of other arboviruses are often only recognised when human or animal cases are diagnosed, a situation exemplified by the regular dengue outbreaks in northern Queensland and the original outbreak of JEV in northern Australia.

The emergence of JEV in northern Australia highlighted a number of unique problems which compromised the implementation of a sustainable surveillance programme to detect future incursions of the virus. This area of northern Australia, encompassing the Torres Strait and Cape York Peninsula is remote, located hundreds of kilometres from diagnostic laboratories. Many of the locations in this region are only accessible by aircraft or boat and wet season rainfall renders most mainland sites inaccessible to road transport between December and May, the period when JEV activity mainly occurs. We describe the history of JEV surveillance in Australia and the development of a novel surveillance system for JEV in remote locations, which also has application for other arboviruses, both within Australia and overseas.

\section{Sentinel Animals for Arbovirus Surveillance}

Sentinel animals have been utilized in Australia since the late 1960s to monitor arbovirus activity [8]. A sentinel animal programme involves placing immunologically naïve animals in a given location, where they are periodically bled and the serum samples submitted for the detection of virus-specific antibodies and/or virus. Sentinel chickens were initially deployed in response to an outbreak of MVEV in southeastern Australia in 1974 [9] and are still employed to detect virus activity in all mainland states $[10,11]$, except Queensland. While sentinel livestock (especially cattle) have been used to detect important veterinary arboviruses, such as bluetongue and bovine ephemeral fever viruses, they have also occasionally seroconverted to MVEV and KUNV [12]. Following the incipient outbreak of JEV on Badu Island in the Torres Strait in 1995, a sentinel pig surveillance system was established to detect further JEV activity in the Torres Strait and determine whether the virus had extended onto Cape York Peninsula on the Australian mainland [13]. In the Torres Strait, this programme was considered successful, detecting JEV in all years (except 1999) between 1996 and 2006.

Despite the ability to detect arbovirus activity, deployment of sentinel animals has a number of drawbacks which compromise their efficacy as a surveillance tool. There are ethical implications associated with using animals. Crossreactions in serological assays make it difficult to distinguish 
closely related viruses, such as JEV and MVEV. Larger animals, such as pigs, can be difficult to bleed, representing an occupational health and safety issue. Some sentinel animals are amplifying hosts of the virus they are deployed to detect (i.e., pigs and JEV), so they may actually contribute to virus transmission cycles. Clearly, an alternative method to sentinel animals needs to be developed for arbovirus surveillance in remote areas.

\section{Mosquito-Based Surveillance of Arboviruses}

In Australia, mosquito-based arbovirus surveillance currently involves collecting mosquitoes in $\mathrm{CO}_{2}$-baited encephalitis vector surveillance (EVS; [14]) or Centers for Disease Control (CDC; [15]) light traps. Once collected, pools of 25-100 individual mosquitoes are processed and a filtered homogenate is inoculated into cell culture and viral antigen is detected with an enzyme-linked immunosorbent assay (ELISA; [16]) or immunofluorescence assay (IFA; [17]). Some Australian states conduct ongoing mosquito trapping for virus isolation, which either runs throughout the year [11] or between November and April [10]. Other states only undertake trapping in response to outbreaks or incursions of virus $[18,19]$. Traps are deployed overnight and mosquitoes collected within 24 hours. Unfortunately, longer periods of deployment are hampered by relatively short battery life necessitating recharging or replacement of batteries, exhaustion of $\mathrm{CO}_{2}$ when dry ice is used, and decreased survival/viability of mosquitoes for extended periods in collection containers. Traps need to be collected within 24 hours and more than 30 traps can be set in a night [11] highlighting the labour-intensive nature of this method of surveillance. This problem is compounded in remote locations, where logistical issues and high costs prevent collection of traps after 24 hours of deployment.

In an attempt to overcome these limitations, a unique system of surveillance based on detecting arboviruses in mosquitoes collected in traps over 7 days has been under development since 2000. This system involves (a) solar-, long life battery-, or propane gas-powered traps, which can run for extended periods; (b) $\mathrm{CO}_{2}$ administered from large capacity compressed gas cylinders; and (c) molecular-based assays which are able to detect viral RNA in mosquitoes which have been held under field conditions for at least 7 days. One trap that showed considerable early potential was the propane-powered Mosquito Magnet (20; Woodstream Corporation, Lititz, PA, USA). This trap functions by combusting propane gas to produce $\mathrm{CO}_{2}$, heat, and moisture as mosquito attractants, while a thermoelectric generator converts excess heat into electricity to power the trap fan [20]. Importantly, a single propane gas cylinder lasts 3 weeks, making this an ideal trap for remote areas. In efficacy trials conducted in northern Australia, the Mosquito Magnet collected at least as many Culex sitiens subgroup mosquitoes (the primary JEV vectors) as the "gold standard" $\mathrm{CO}_{2}$-baited Centers for Disease Control light trap [21].
Since virus degradation after 7 days under tropical conditions was to be expected, molecular methods for viral RNA detection were tested for sensitivity. Preservation of infectious virus between remote trapping location and laboratory depends on a cold chain which is difficult to maintain. A number of laboratory-based experiments demonstrated that RT-PCR detected WNV, St. Louis encephalitis virus, western equine encephalitis virus, and DENV RNA in mosquitoes stored at room temperature or under simulated conditions of high temperature and humidity [22-24]. PCRbased detection methods were able to detect JEV RNA in a single mosquito in pools of up to 1,000 uninfected mosquitoes stored for at least 14 days under simulated tropical conditions [25]. Similarly, during a field trial in Cairns, northern Australia, single laboratory-infected mosquitoes were detected in pools of up to 1,000 mosquitoes stored for 14 days within a functioning Mosquito Magnet [26]. Furthermore, RT-PCR was used to detect DENV-2 RNA in laboratory-infected mosquitoes adhered to sticky traps set under natural conditions of high heat and humidity [27], while DENV-3 RNA was detected in 6 pools of Ae. aegypti removed from sticky ovitraps deployed during an outbreak in Cairns [28].

Between 2001 and 2005, a field trial was conducted in the Torres Strait and northern Cape York Peninsula with the objective of comparing the mosquito-based surveillance system with the sentinel pig programme [29]. Weekly mosquito collections from either the Mosquito Magnet and/or the Northern Australian Quarantine Strategy Mozzie Trap (NMT; 29), a trap developed for the purposes of long term deployment, were submitted for detection of JEV RNA. Sentinel pigs were bled weekly and serum samples were submitted for detection of JEV-specific antibodies by ELISA or viral RNA by real-time TaqMan RT-PCR [30]. The mosquito-based system successfully detected JEV and demonstrated the feasibility of the concept. However, it did not detect JEV before detection in the sentinel pigs on either Badu Island or St. Pauls community on Moa Island and did not detect virus on the mainland. There were a number of other logistical issues with the mosquitobased system. Firstly, the collection of large numbers of non-target Aedes spp. congested fans and necessitated presorting of collections. Indeed, a single weekly Mosquito Magnet collection yielded $>178,000$ mosquitoes of which $<1 \%$ were $C x$. sitiens subgroup mosquitoes. The number of non-target species was significantly reduced when 1octen-3-ol (octenol), a chemical used to increase collections of some mosquito species, was removed from the traps [31]. A lack of ventilation, especially in the NMT, caused considerable bacterial and fungal contamination of collected mosquitoes, which may have led to degradation of viral RNA and therefore reduced the ability to detect viral RNA by TaqMan RT-PCR. The hot, humid conditions coupled with a lack of regular maintenance led to blocked gas lines and component malfunction, which decreased the efficiency of both types of traps. Finally, the system was determined to be insufficiently sensitive, as it was estimated that over 47,000 $C x$. sitiens subgroup mosquitoes would have to be processed from the mainland for a single JEV detection, equating to 114 


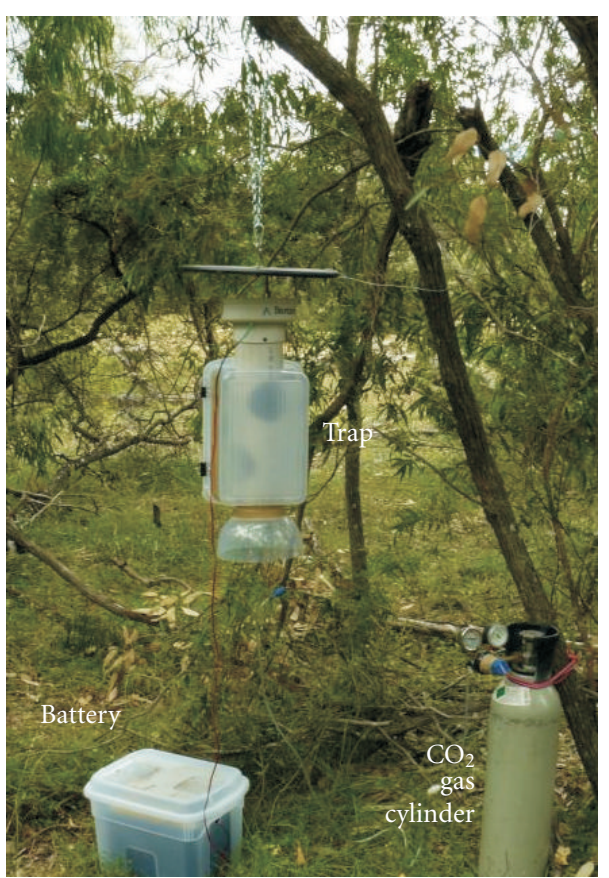

(a)

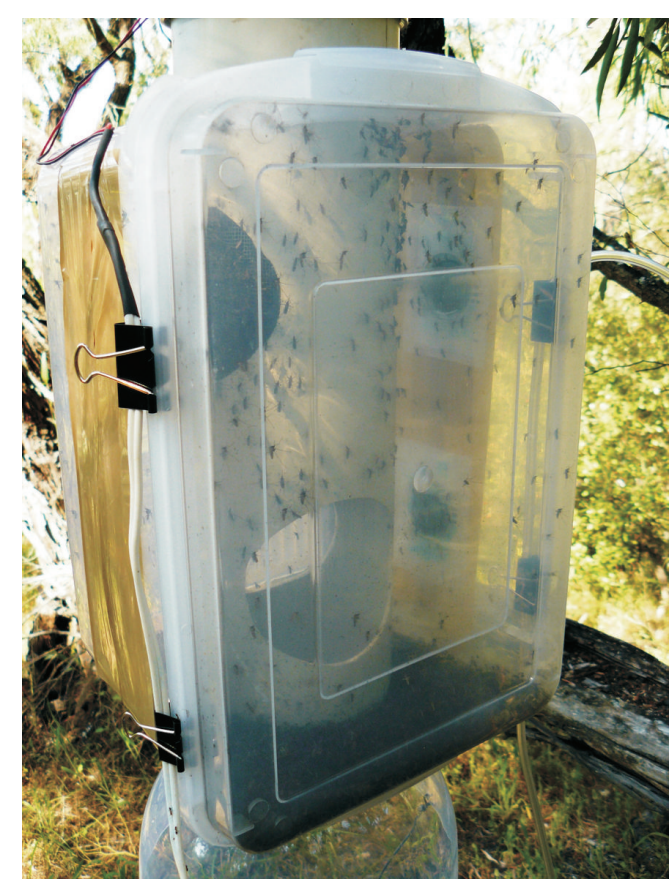

(b)

Figure 2: $\mathrm{CO}_{2}$-baited updraft box trap deployed near Bunbury, Western Australia. (a) The trap is powered by a battery and baited with $\mathrm{CO}_{2}$ released from a compressed gas cylinder. (b) Close-up view of trap showing collected mosquitoes (photographs courtesy of Scott Dandridge).

trap nights [29]. Although the results from the field trial were promising, the issues identified during the trial prevented mosquito-based surveillance on a large scale as a replacement for sentinel animals.

\section{Development of a Mosquito-Free Arbovirus Surveillance System}

To circumvent some of the issues listed previously, particularly the need to process large numbers of mosquitoes, a strategy was developed whereby collected mosquitoes probe a substrate, and the substrate, not the mosquitoes, is submitted for virus detection. It was originally suggested by Doggett and others that virus could be transferred from infected to uninfected mosquitoes while feeding from the same sucrose soaked cotton pledget [32]. However, they were unable to detect virus on the sucrose pledget using a fixed cell culture ELISA. Based on these observations, a series of experiments utilising TaqMan RT-PCR was conducted to assess whether mosquitoes expectorate viruses when they sugar feed [33]. Not only were JEV, MVEV, and KUNV RNA detected in pledgets removed from batches of up to 50 infected mosquitoes, but also JEV RNA was detected in $73 \%$ of pledgets that had been exposed to individual infected mosquitoes [33].

The discovery that mosquitoes expectorate virus when they sugar feed formed the basis for a "mosquito-free" surveillance system. In this system, mosquitoes are attracted to a $\mathrm{CO}_{2}$-baited trap, which possesses a holding container where mosquitoes are provided access to a sugar-baited substrate [34]. Several substrates were tested in the laboratory and it was found that Flinders Technology Associates (FTA; Whatman International Ltd, Maidstone, UK) cards could bind and preserve viral RNA for at least 28 days, inactivate virus on contact for safer handling, and resist bacterial and fungal contamination. Honey was chosen as the sugar source, because it remains moist on the FTA cards for at least a week and honey contains antibacterial properties which would also limit degradation of viral RNA [34-36]. Importantly, CHIKV, KUNV, and RRV RNA could be detected on $>70 \%$ of honey-baited FTA cards that had been fed upon by infected mosquitoes.

In parallel with the laboratory-based experiments, a $\mathrm{CO}_{2}$-baited updraft box trap which collected and housed mosquitoes was developed and tested. This trap utilized updraft technology [37] and incorporated a motorised fan which draws mosquitoes into a collection container where mosquitoes could access the honey-baited FTA cards. In preliminary field trials, the updraft box trap collected more mosquitoes than a $\mathrm{CO}_{2}$-baited $\mathrm{CDC}$ trap and between $77-95 \%$ of collected mosquitoes fed on the honey-baited substrates [34].

The final step in the development of the "mosquito-free" surveillance system was to test the efficacy of the system for detection of arboviruses in a field setting [34]. Trials were undertaken during 2008 and 2009 at locations where RRV and BFV activity historically occurred near Bunbury, southwestern Western Australia, and Cairns (Figure 2). At each location, two updraft box traps were set, with each trap 
containing 4-6 honey-soaked FTA cards. Traps were serviced weekly and the FTA cards and mosquitoes were sent to the laboratory for detection of viral RNA using TaqMan RTPCR. During the trials, RRV and BFV RNA was detected in both FTA cards and mosquito pools [34].

Although the efficacy of the honey-baited system of arbovirus surveillance had been demonstrated under field conditions, its sensitivity compared to sentinel animals had yet to be evaluated. Consequently, a field trial was conducted during the 2009-2010 wet season with the objective to compare the honey-bait system and sentinel chickens for the detection of MVEV or KUNV activity at Kununurra in the remote north of Western Australia. The honey-soaked cards were submitted for virus detection using TaqMan RT-PCR and the chicken sera tested for virus-specific antibodies in a modified blocking ELISA [38]. Unfortunately, during the 14-week trial, there was no evidence of flavivirus activity in either the sentinel chickens or the honey-baited FTA cards, which was only the second time in over 20 years that MVEV or KUNV activity was not detected at the study location. A field comparison of the honey-bait system and a sentinel animal system is still to be conducted during a period of recognized arbovirus activity.

\section{New Directions for Mosquito Collection}

All of the mosquito traps described previously utilise various combinations of $\mathrm{CO}_{2}$ and light to attract mosquitoes to the trap, and battery-powered fans to draw attracted mosquitoes into a collection bag or chamber. However, access to electricity for powering traps can be a significant hurdle for remote trapping and hot humid conditions can damage motorised components. Even though the Mosquito Magnet traps utilised combustion of propane to power the fan, as well as create heat and $\mathrm{CO}_{2}$ to attract mosquitoes, the issues with dependability meant that it was not practical for use in remote locations.

Recently, we have developed a passive nonmechanical fanless trap for collection of mosquitoes in the honeybait surveillance system. This trap extends work that was conducted over 40 years ago by Schreck and others who developed a passive trap consisting of a plexiglass box with screened cone entry points that collected large numbers of mosquitoes [39]. Our trap consists of a translucent plastic crate connected via a hose to an external $\mathrm{CO}_{2}$ source. Mosquitoes enter the trap through the bottom of the crate, attracted by the $\mathrm{CO}_{2}$. Once inside, the mosquitoes are attracted to the outdoor light transmitted through the sides of the translucent crate, helping to retain mosquitoes inside where they feed on honey-soaked FTA cards. In field trials in Cairns, Australia, and Florida, USA, passive traps baited with $\mathrm{CO}_{2}$ from dry ice collected $185 \%$ and $50 \%$ of the number of mosquitoes, respectively, as a $\mathrm{CO}_{2}$-baited $\mathrm{CDC}$ light trap (S. Ritchie, G. Cortis and D. Shroyer, unpublished data). As mosquitoes escaping from the passive trap were observed in the Florida trial (D. Shroyer, personal communication), further refinements are needed to maximize mosquito capture and retention.
Finding a suitable $\mathrm{CO}_{2}$ source to attract mosquitoes in remote locations poses many problems. Dry ice is impractical for long-term trapping. When using compressed gas cylinders as a $\mathrm{CO}_{2}$ source, issues such as expense, heavy weight, requirement for specialised regulators, and transport as dangerous goods need to be considered. A relatively simple and inexpensive system that offers great potential as a $\mathrm{CO}_{2}$ source is the production of $\mathrm{CO}_{2}$ during fermentation reactions involving yeast, sugar, and water [40, 41]. Such fermentation-derived $\mathrm{CO}_{2}$ could be greatly improved by the development of yeast strains that produce high levels of $\mathrm{CO}_{2}$ at lower temperatures, and storage systems that only release $\mathrm{CO}_{2}$ when vectors are active, for example, at night.

The addition of semiochemicals that work either alone or synergistically with $\mathrm{CO}_{2}$ could also be used to increase collections of hematophagous insects including mosquitoes $[42,43]$. When used as an attractant in traps, these lures significantly increase the collection of mosquitoes such as Ae. albopictus and Anopheles gambiae [44, 45]. One chemical that has received much attention is octenol which, when added to $\mathrm{CO}_{2}$ in light traps, could significantly increase collections of important vectors of RRV [46] and JEV [31], as well as other biting flies, including culicoides [47] and phlebotomines [48]. However, due to intraspecific differences in responses to octenol [31], preliminary trials should be conducted to determine the suitability for its use as an added attractant.

The $\mathrm{CO}_{2}$-baited passive traps could be modified into simplified "killing traps" that not only facilitate detection of arboviruses but also kill attracted mosquitoes. The honey bait could be laced with nonrepellent, rapid-acting insecticides such as bendiocarb [49] or imidacloprid [50]. Because mosquitoes do not need to be retained in the trap, it can be more open, increasing mosquito access to the honeysoaked FTA cards and to insecticides, thus increasing killing power. Furthermore, nonrepellent insecticides can be used to treat the interior of the box trap or vaporized, nonrepellent insecticides such as dichlorvos and metofluthrin [51] can be placed inside the box to kill attracted mosquitoes. A perimeter line of surveillance and killing traps could be used to maximize surveillance while providing limited control. This trap line could consist of conduit connecting a large $\mathrm{CO}_{2}$ source to several trap units, similar to the lure and kill method used to control Ae. taeniorhynchus at a resort near Naples, Florida [42].

Modern sensor technology and data transfer systems could be harnessed to create remote mosquito sensors and traps that notify users of not only the magnitude and identity of mosquitoes sensed but also possible infection with arboviruses on honey-soaked cards. Indeed, sensors that detect and identify mosquitoes by wing-beat frequency have been developed and teamed with lasers to track and kill detected mosquitoes (http://intellectualventureslab.com/?page_id=563). Acoustic signals could be used to estimate the number of mosquitoes within a trap, and biosensor systems [52] could be utilized to identify arboviruses expectorated onto honey-baited cards. 


\section{New Directions for Detection of Virus in Substrates or Mosquitoes}

The sensitive detection of the viruses obtained by surveillance is a crucial aspect of any methodology. Furthermore, there has been a recent emergence and re-emergence of viral pathogens such as JEV, WNV, CHIKV, Usutu virus, and Alkhurma virus, so testing routinely for exotic and new viruses becomes an important component of surveillance to protect both human and animal health. However, many of the molecular methods for detection of arboviruses are limited to characterized viruses with specific primer and probe sets.

Testing for novel viruses without prior knowledge of the pathogen has become possible with new high throughput sequencing methods, also known as next generation sequencing (NGS). They enable the rapid and sensitive detection of large numbers of known and unknown viruses in a sample. Various chemistries for NGS are available and continually being developed, but most perform a reaction with a template clonally amplified on microscopic beads (reviewed in [53]). The combined outputs from hundreds of thousands or even millions of such beads generate enormous amounts of sequencing data. The generated data is independent of sequence, so that any nucleic acid in a sample is a potential template and therefore it by-passes the challenges associated with the detection of viruses that cannot be cultured in the laboratory. Using NGS, the potential exists for the discovery of exotic and new viruses. In addition, software tools being developed for the related field of microbial metagenomics [54], or the study of microbial communities using primarily NGS methods, will facilitate the analysis of the large amounts of sequence data generated from samples obtained in the honey-bait surveillance system.

Like any new technology, there are some drawbacks to NGS. It is currently relatively expensive in comparison with traditional detection methods, although the cost per base sequenced is decreasing rapidly $[55,56]$. Some potential also exists for bias introduced by the nucleic acid amplification steps of the methods [57], and this should be a consideration during experimental design. Nonetheless, in support of their application to arbovirus surveillance, NGS and metagenomics have been successfully applied to the surveillance of viruses in bat communities [58,59], in fermented food [60], and in human clinical samples [6163]. There is considerable potential for this technology to identify new arboviral threats to human and animal health, to examine how arbovirus populations change with time, and to reveal how environmental factors affect the emergence of new strains and influence spread from animal reservoirs into human populations.

\section{Conclusions}

Effective surveillance forms a vital component of any programme aimed at reducing the impact of arboviruses on human and animal health. A multidisciplinary, holistic system is the best approach. It could incorporate the latest scientific advances such as NGS for virus detection, sophisticated surveillance tools such as biosensors to collect data on mosquitoes and viruses, meteorological data, and production of effective, low-cost, nonmechanical traps coupled with yeast-generated $\mathrm{CO}_{2}$. Such uncomplicated traps have the greatest applicability for deployment in remote locations. The information obtained from such a system could then be uploaded for dissemination to end users employing data sharing technology, such as a "cloud computing" platform [64]. Arbovirus surveillance data in north-eastern Australia has been erratic in the past mainly due to its remoteness. The simplicity of using the described traps and the honey-baited FTA cards together with advancing information technology would ensure continuous collection of surveillance data.

Even though vector species identification and infection rates in mosquito populations cannot be determined using the honey-bait system, it can provide an early warning of impending virus activity, in much the same way as a sentinel animal programme. Disease control strategies, such as mosquito control, vaccination, or health promotion initiatives, can then be implemented. Focussed trapping can also be undertaken to incriminate vector species, calculate virus carriage rates, as well as determine important entomological characteristics that can influence transmission, including host feeding patterns and the genetic structure of the vector populations.

\section{Acknowledgments}

The studies described in the current paper would not have been possible without the assistance and advice of a great many people, particularly Giles Cortis, Roy Hall, Paul Zborowski, Alyssa Pyke, Greg Smith, Ian Walsh, Brian Montgomery, Stephen Frances, Bob Cooper, Cassie Jansen, Petrina Johnson, Joe Davis, David Banks, Scott Dandridge, Donald Shroyer, and John Mackenzie. The authors also thank the staff of the Northern Australia Quarantine Strategy for assistance with trapping and staff of Public Health Virology, Queensland Health Forensic and Scientific Services, for assistance with the analysis of samples. This work was funded by the Australian Health Ministers Advisory Council Priority Driven Research Program, the Australian Biosecurity Cooperative Research Centre for Emerging Infectious Disease, and Queensland Health.

\section{References}

[1] R. C. Russell and D. E. Dwyer, "Arboviruses associated with human disease in Australia," Microbes and Infection, vol. 2, no. 14, pp. 1693-1704, 2000.

[2] G. J. Fitzsimmons, P. Wright, C. A. Johansen, P. I. Whelan, and National Arbovirus and Malaria Advisory Committee, "Arboviral diseases and malaria in Australia, 2007/08: annual report of the National Arbovirus and Malaria Advisory Committee," Communicable Diseases Intelligence, vol. 33, no. 2, pp. 155-169, 2009.

[3] J. N. Hanna and S. A. Ritchie, "Outbreaks of dengue in north Queensland, 1990-2008," Communicable Diseases Intelligence, vol. 33, no. 1, pp. 32-33, 2009. 
[4] J. N. Hanna, S. A. Ritchie, D. A. Phillips et al., "An outbreak of Japanese encephalitis in the Torres Strait, Australia, 1995," Medical Journal of Australia, vol. 165, no. 5, pp. 256-260, 1996.

[5] C. C. Jansen, C. E. Webb, J. A. Northill, S. A. Ritchie, R. C. Russell, and A. F. van den Hurk, "Vector competence of Australian mosquito species for a North American strain of West Nile virus," Vector-Borne and Zoonotic Diseases, vol. 8, no. 6, pp. 805-811, 2008.

[6] M. J. Turell and B. H. Kay, "Susceptibility of selected strains of Australian mosquitoes (Diptera: Culicidae) to rift valley fever virus," Journal of Medical Entomology, vol. 35, no. 2, pp. 132135, 1998.

[7] A. F. van den Hurk, S. Hall-Mendelin, A. T. Pyke, G. A. Smith, and J. S. Mackenzie, "Vector competence of Australian mosquitoes for chikungunya virus," Vector-Borne and Zoonotic Diseases, vol. 10, no. 5, pp. 489-495, 2010.

[8] T. D. S. George, "A sentinel herd system for the study of arbovirus infections in Australia and Papua-New Guinea," Veterinary Science Communications, vol. 4, no. 1, pp. 39-51, 1980.

[9] J. Campbell and D. E. Hore, "Isolation of Murray Valley encephalitis virus from sentinel chickens," Australian Veterinary Journal, vol. 51, no. 1, pp. 1-3, 1975.

[10] S. Doggett, J. Clancy, J. Haniotis et al., The New South Wales Arbovirus Surveillance and Mosquito Monitoring Program 2010-2011 Annual Report, Department of Medical Entomology, ICPMR, Westmead Hospital, Westmead, Australia, 2011.

[11] C. Johansen, S. McFall, S. Wong et al., The University of Western Australia Arbovirus Surveillance and Research Laboratory Annual Report Summary: 2009-2010, University of Western Australia, Perth, Australia, 2010.

[12] P. I. Whelan and G. P. Gard, "Arbovirus surveillance in the Northern Territory 1981," Communicable Diseases Intelligence, vol. 12, no. 1, pp. 2-3, 1981.

[13] J. Shield, J. Hanna, and D. Phillips, "Reappearance of the Japanese encephalitis virus in the Torres Strait, 1996," Communicable Diseases Intelligence, vol. 20, no. 8, p. 191, 1996.

[14] D. Rohe and R. P. Fall, "A miniature battery powered $\mathrm{CO}_{2}$ baited light trap for mosquito borne encephalitis surveillance," Bulletin of the Society for Vector Ecology, vol. 4, no. 1, pp. 24-27, 1979.

[15] W. D. Sudia and R. W. Chamberlain, "Battery-operated light trap, an improved model," Mosquito News, vol. 22, no. 2, pp. 126-129, 1962.

[16] A. K. Broom, R. A. Hall, C. A. Johansen et al., "Identification of Australian arboviruses in inoculated cell cultures using monoclonal antibodies in ELISA," Pathology, vol. 30, no. 3, pp. 286-288, 1998.

[17] B. Forghani and S. Hagens, "Diagnosis of viral infections by antigen detection," in Diagnostic Procedures for Viral, Rickettsial and Chlamydial Infections, E. H. Lennette, D. A. Lennette, and E. T. Lennette, Eds., pp. 79-96, American Public Health Association, Washington, DC, USA, 7th edition, 1995.

[18] S. A. Ritchie, I. D. Fanning, D. A. Phillips, H. A. Standfast, D. McGinn, and B. H. Kay, "Ross River virus in mosquitoes (Diptera: Culicidae) during the 1994 epidemic around Brisbane, Australia," Journal of Medical Entomology, vol. 34, no. 2, pp. 156-159, 1997.

[19] S. A. Ritchie, D. Phillips, A. Broom, J. Mackenzie, M. Poidinger, and A. van den Hurk, "Isolation of Japanese encephalitis virus from Culex annulirostris in Australia," American Journal of Tropical Medicine and Hygiene, vol. 56, no. 1, pp. 80-84, 1997.
[20] D. L. Kline, "Evaluation of various models of propanepowered mosquito traps," Journal of Vector Ecology, vol. 27, no. 1, pp. 1-7, 2002.

[21] C. A. Johansen, B. L. Montgomery, J. S. Mackenzie, and S. A. Ritchie, "Efficacies of the MosquitoMagnet and counterflow geometry traps in north Queensland, Australia," Journal of the American Mosquito Control Association, vol. 19, no. 3, pp. 265270, 2003.

[22] M. J. Bangs, R. Tan, E. Listiyaningsih, B. H. Kay, and K. R. Porter, "Detection of dengue viral RNA in Aedes aegypti (diptera: culicidae) exposed to sticky lures using reversetranscriptase polymerase chain reaction," Journal of Medical Entomology, vol. 38, no. 5, pp. 720-724, 2001.

[23] L. D. Kramer, R. E. Chiles, T. Do, and H. M. Fallah, "Detection of St. Louis encephalitis and western equine encephalomyelitis RNA in mosquitoes tested without maintenance of a cold chain," Journal of the American Mosquito Control Association, vol. 17, no. 4, pp. 213-215, 2001.

[24] M. J. Turell, A. R. Spring, M. K. Miller, and C. E. Cannon, "Effect of holding conditions on the detection of West Nile viral RNA by reverse transcriptase-polymerase chain reaction from mosquito (Diptera: Culicidae) pools," Journal of Medical Entomology, vol. 39, no. 1, pp. 1-3, 2002.

[25] C. A. Johansen, R. A. Hall, A. F. van den Hurk, S. A. Ritchie, and J. S. Mackenzie, "Detection and stability of Japanese encephalitis virus RNA and virus viability in dead infected mosquitoes under different storage conditions," American Journal of Tropical Medicine and Hygiene, vol. 67, no. 6, pp. 656-661, 2002.

[26] S. A. Ritchie, A. T. Pyke, G. A. Smith et al., "Field evaluation of a sentinel mosquito (Diptera: Culicidae) trap system to detect Japanese encephalitis in remote Australia," Journal of Medical Entomology, vol. 40, no. 3, pp. 249-252, 2003.

[27] M. J. Bangs, R. Pudiantari, and Y. R. Gionar, "Persistence of dengue virus RNA in dried Aedes aegypti (Diptera: Culicidae) exposed to natural tropical conditions," Journal of Medical Entomology, vol. 44, no. 1, pp. 163-167, 2007.

[28] S. A. Ritchie, S. Long, G. Smith, A. Pyke, and T. B. Knox, "Entomological investigations in a focus of dengue transmission in Cairns, Queensland, Australia, by using the sticky ovitraps," Journal of Medical Entomology, vol. 41, no. 1, pp. 14, 2004.

[29] S. A. Ritchie, A. F. van den Hurk, P. Zborowski et al., "Operational trials of remote mosquito trap systems for Japanese encephalitis virus surveillance in the Torres Strait, Australia," Vector-Borne and Zoonotic Diseases, vol. 7, no. 4, pp. 497-506, 2007.

[30] A. T. Pyke, I. L. Smith, A. F. van den Hurk et al., "Detection of Australasian Flavivirus encephalitic viruses using rapid fluorogenic TaqMan RT-PCR assays," Journal of Virological Methods, vol. 117, no. 2, pp. 161-167, 2004.

[31] A. F. van den Hurk, B. L. Montgomery, P. Zborowski, N. W. Beebe, R. D. Cooper, and S. A. Ritchie, "Does 1octen-3-ol enhance trap collections of Japanese encephalitis virus mosquito vectors in northern Australia?" Journal of the American Mosquito Control Association, vol. 22, no. 1, pp. 1521, 2006.

[32] S. L. Doggett, M. J. Klowden, and R. C. Russell, "Are vector competence experiments competent vector experiments?" Arbovirus Research in Australia, vol. 8, pp. 126-130, 2001.

[33] A. F. van den Hurk, P. H. Johnson, S. Hall-Mendelin et al., "Expectoration of flaviviruses during sugar feeding by mosquitoes (Diptera: Culicidae)," Journal of Medical Entomology, vol. 44, no. 5, pp. 845-850, 2007. 
[34] S. Hall-Mendelin, S. A. Ritchie, C. A. Johansen et al., "Exploiting mosquito sugar feeding to detect mosquito-borne pathogens," Proceedings of the National Academy of Sciences of the United States of America, vol. 107, no. 25, pp. 11255-11259, 2010.

[35] P. E. Lusby, A. L. Coombes, and J. M. Wilkinson, "Bactericidal activity of different honeys against pathogenic bacteria," Archives of Medical Research, vol. 36, no. 5, pp. 464-467, 2005.

[36] R. J. Weston, L. K. Brocklebank, and Y. Lu, "Identification and quantitative levels of antibacterial components of some New Zealand honeys," Food Chemistry, vol. 70, no. 4, pp. 427-435, 2000.

[37] S. A. Ritchie, P. Zborowski, D. Banks, I. Walsh, and J. Davis, "Efficacy of novel updraft traps for collection of mosquitoes in Cairns, Australia," Journal of the American Mosquito Control Association, vol. 24, no. 4, pp. 520-527, 2008.

[38] R. A. Hall, A. K. Broom, A. C. Hartnett, M. J. Howard, and J. S. Mackenzie, "Immunodominant epitopes on the NS1 proteins of MVE and KUN viruses serve as targets for a blocking ELISA to detect virus-specific antibodies in sentinel animal serum," Journal of Virological Methods, vol. 51, no. 2-3, pp. 201-210, 1995.

[39] C. E. Schreck, H. K. Gouck, and K. H. Posey, "An experimental Plexiglas mosquito trap utilizing carbon dioxide," Mosquito News, vol. 30, no. 4, pp. 641-645, 1970.

[40] Y. Saitoh, J. Hattori, S. Chinone et al., "Yeast-generated $\mathrm{CO}_{2}$ as a convenient source of carbon dioxide for adult mosquito sampling," Journal of the American Mosquito Control Association, vol. 20, no. 3, pp. 261-264, 2004.

[41] R. C. Smallegange, W. H. Schmied, K. J. Van Roey et al., "Sugar-fermenting yeast as an organic source of carbon dioxide to attract the malaria mosquito Anopheles gambiae," Malaria Journal, vol. 9, no. 1, article no. 292, 2010.

[42] D. L. Kline, "Traps and trapping techniques for adult mosquito control," Journal of the American Mosquito Control Association, vol. 22, no. 3, pp. 490-496, 2006.

[43] D. L. Kline, "Semiochemicals, traps/targets and mass trapping technology for mosquito management," Journal of the American Mosquito Control Association, vol. 23, supplement 2, pp. 241-251, 2007.

[44] D. F. Hoel, D. L. Kline, S. A. Allan, and A. Grant, "Evaluation of carbon dioxide, 1-octen-3-ol, and lactic acid as baits in Mosquito Magnet Pro traps for Aedes albopictus in north central Florida," Journal of the American Mosquito Control Association, vol. 23, no. 1, pp. 11-17, 2007.

[45] F. O. Okumu, G. F. Killeen, S. Ogoma et al., "Development and field evaluation of a synthetic mosquito lure that is more attractive than humans," PloS One, vol. 5, no. 1, p. e8951, 2010.

[46] J. A. Kemme, P. H. Van Essen, S. A. Ritchie, and B. H. Kay, "Response of mosquitoes to carbon dioxide and 1-octen-3-ol in southeast Queensland, Australia," Journal of the American Mosquito Control Association, vol. 9, no. 4, pp. 431-435, 1993.

[47] S. A. Ritchie, P. H. van Essen, J. A. Kemme, B. H. Kay, and D. Allaway, "Response of biting midges (Diptera: Ceratopogonidae) to carbon dioxide, octenol, and light in southeastern Queensland, Australia," Journal of Medical Entomology, vol. 31, no. 5, pp. 645-648, 1994.

[48] G. M. Beavers, H. A. Hanafi, and E. A. Dykstra, "Evaluation of 1-octen-3-ol and carbon dioxide as attractants for Phlebotomus papatasi (Diptera: Psychodidae) in southern Egypt," Journal of the American Mosquito Control Association, vol. 20, no. 2, pp. 130-133, 2004.

[49] R. G. Evans, "Laboratory evaluation of the irritancy of bendiocarb, lambda-cyhalothrin and DDT to Anopheles gambiae,"
Journal of the American Mosquito Control Association, vol. 9, no. 3, pp. 285-293, 1993.

[50] W. H. White, C. M. McCoy, J. A. Meyer et al., "Knockdown and mortality comparisons among spinosad-, imidacloprid, and methomyl-containing baits against susceptible Musca domestica (Diptera: Muscidae) under laboratory conditions," Journal of Economic Entomology, vol. 100, no. 1, pp. 155-163, 2007.

[51] L. P. Rapley, R. C. Russell, B. L. Montgomery, and S. A. Ritchie, "The effects of sustained release metofluthrin on the biting, movement, and mortality of Aedes aegypti in a domestic setting," American Journal of Tropical Medicine and Hygiene, vol. 81, no. 1, pp. 94-99, 2009.

[52] F. S. Teles, "Biosensors and rapid diagnostic tests on the frontier between analytical and clinical chemistry for biomolecular diagnosis of dengue disease: a review," Analytica Chimica Acta, vol. 687, no. 1, pp. 28-42, 2011.

[53] M. Kircher and J. Kelso, "High-throughput DNA sequencing-concepts and limitations," BioEssays, vol. 32, no. 6, pp. 524-536, 2010.

[54] D. H. Huson, A. F. Auch, J. Qi, and S. C. Schuster, "MEGAN analysis of metagenomic data," Genome Research, vol. 17, no. 3, pp. 377-386, 2007.

[55] T. W. Scott, S. A. Wright, B. F. Eldridge, and D. A. Brown, "Cost effectiveness of three arbovirus surveillance methods in northern California," Journal of the American Mosquito Control Association, vol. 17, no. 2, pp. 118-123, 2001.

[56] L. Bonetta, "Whole-Genome sequencing breaks the cost barrier," Cell, vol. 141, no. 6, pp. 917-919, 2010.

[57] K.-H. Kim and J.-W. Bae, "Amplification methods bias metagenomic libraries of uncultured single-stranded and double-stranded DNA viruses," Applied and Environmental Microbiology, vol. 77, no. 21, pp. 7663-7668, 2011.

[58] E. F. Donaldson, A. N. Haskew, J. E. Gates, J. Huynh, C. J. Moore, and M. B. Frieman, "Metagenomic analysis of the viromes of three North American bat species: viral diversity among different bat species that share a common habitat," Journal of Virology, vol. 84, no. 24, pp. 13004-13018, 2010.

[59] L. Li, J. G. Victoria, C. Wang et al., "Bat guano virome: predominance of dietary viruses from insects and plants plus novel mammalian viruses," Journal of Virology, vol. 84, no. 14, pp. 6955-6965, 2010.

[60] E.-J. Park, K.-H. Kim, G. C. J. Abell, M.-S. Kim, S. W. Roh, and J.-W. Bae, "Metagenomic analysis of the viral communities in fermented foods," Applied and Environmental Microbiology, vol. 77, no. 4, pp. 1284-1291, 2011.

[61] D. Bogaert, B. Keijser, S. Huse et al., "Variability and diversity of nasopharyngeal microbiota in children: a metagenomic analysis," PLoS One, vol. 6, no. 2, Article ID e17035, 2011.

[62] A. L. Greninger, E. C. Chen, T. Sittler et al., "A metagenomic analysis of pandemic influenza a $(2009 \mathrm{H} 1 \mathrm{~N} 1)$ infection in patients from North America," PLoS One, vol. 5, no. 10, Article ID e13381, 2010.

[63] S. Nakamura, C. S. Yang, N. Sakon et al., "Direct metagenomic detection of viral pathogens in nasal and fecal specimens using an unbiased high-throughput sequencing approach," PLoS One, vol. 4, no. 1, Article ID e4219, 2009.

[64] M. C. Schatz, B. Langmead, and S. L. Salzberg, "Cloud computing and the DNA data race," Nature Biotechnology, vol. 28, no. 7, pp. 691-693, 2010. 

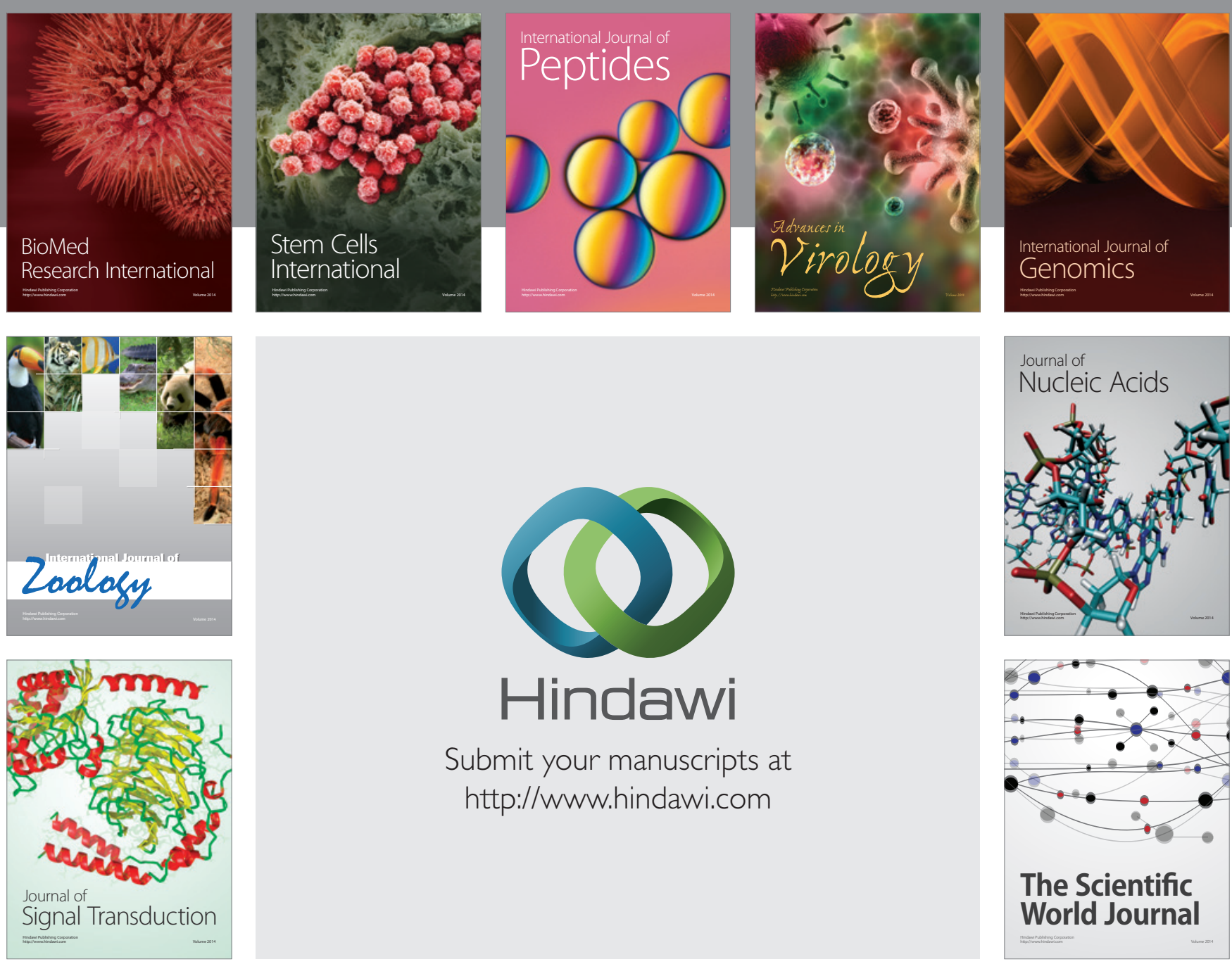

Submit your manuscripts at

http://www.hindawi.com
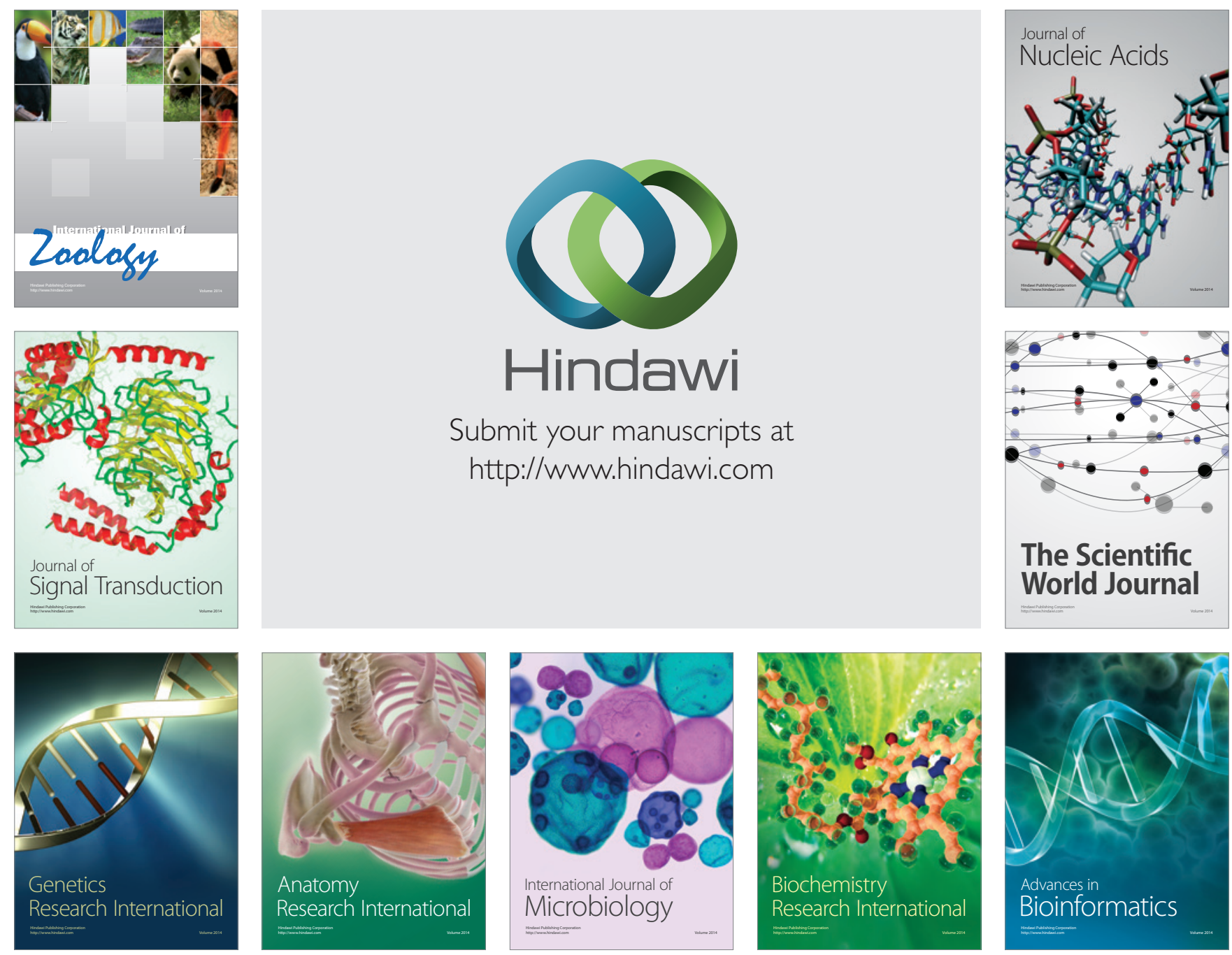

The Scientific World Journal
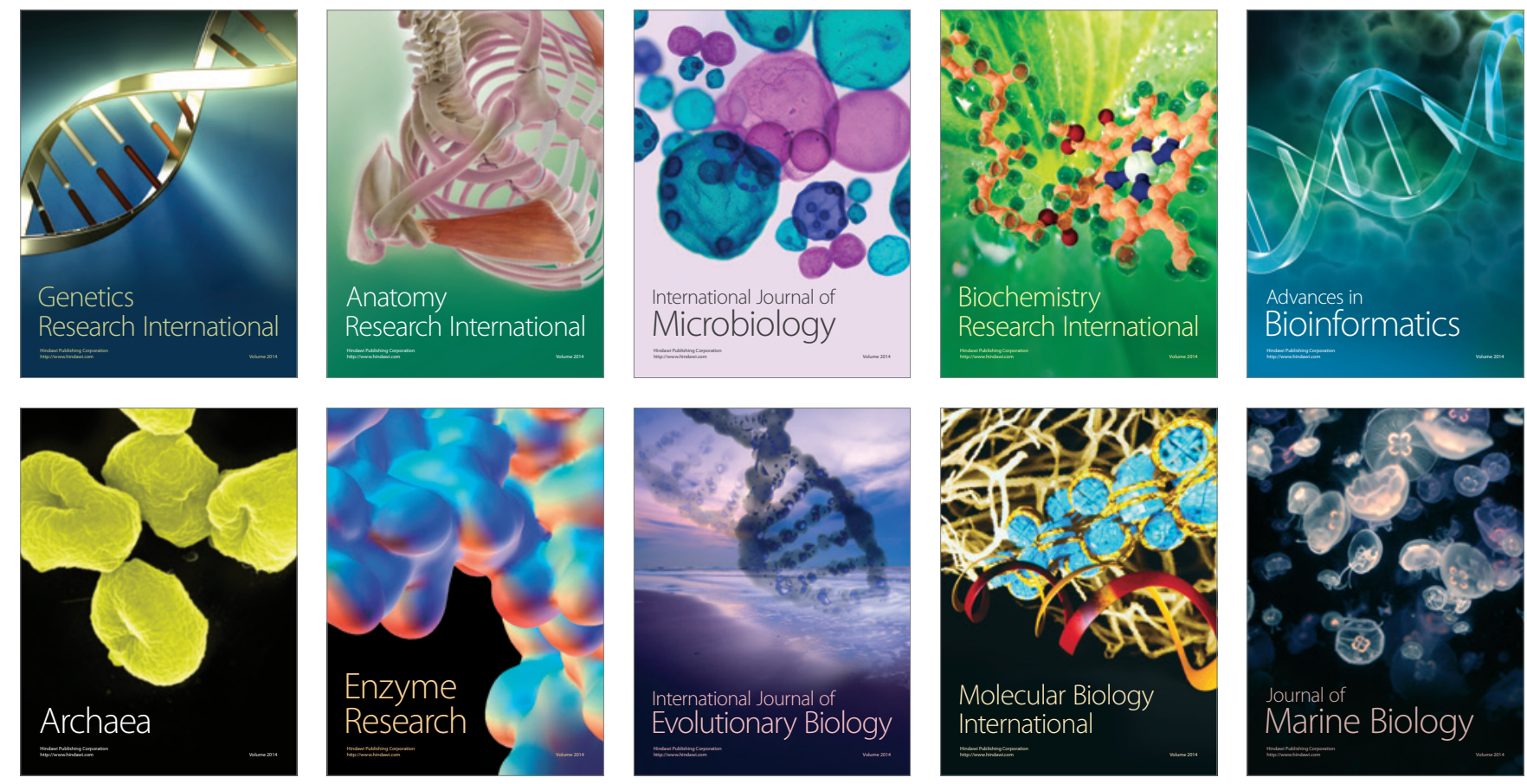